\title{
ANÁLISE FLUIDODINÂMICA COMPUTACIONAL DE ESCOAMENTO PULSÁTIL EM ANEURISMA
}

\author{
Y. C. BOITE ${ }^{1}$, E. WAJNBERG ${ }^{2}$, T. S. KLEIN ${ }^{1}$, R. MEDRONHO $^{1}$ \\ ${ }^{1}$ Universidade Federal do Rio de Janeiro, Escola de Química, Lab CFD \\ ${ }^{2}$ Américas Serviços Médicos - Hospital Vitória \\ E-mail para contato: yasmincarvalho13@ hotmail.com
}

\begin{abstract}
RESUMO - A influência de variáveis hemodinâmicas no crescimento e ruptura de aneurismas tem sido crescentemente investigada com o uso de fluidodinâmica computacional. Perfis de escoamento e da tensão cisalhante são destacados na literatura como sinalizadores de ruptura. Neste trabalho estes parâmetros foram analisados através de simulações CFD em um aneurisma sacular localizado na artéria cerebral média (MCA) utilizando a geometria real de um paciente. Mesmo com a utilização de condições de contorno não específicas do paciente, os resultados mostram corroborar com a literatura.
\end{abstract}

\section{INTRODUÇÃO}

Apesar de se desconhecer os mecanismos exatos que levam à formação, crescimento e ruptura de aneurismas, sabe-se que estão relacionados à hemodinâmica (Dempere-Marco et al., 2006; Mut et al., 2011). Neste contexto, o uso de Fluidodinâmica computacional (CFD) para investigação destes fatores tem crescido. Diferentes estudos buscam validar o uso desta técnica e avaliar quais variáveis hemodinâmicas estão mais intimamente ligadas ao risco de ruptura. Sforza et al. (2016), Mut et al. (2011) e Xiang et al., (2011) concluíram que a presença de múltiplos vórtices e de escoamentos complexos dentro do aneurisma assim como uma tensão cisalhante elevada concentrada em pequenas áreas estão relacionados a um alto risco de ruptura. Portanto, neste trabalho, buscou-se avaliar estes parâmetros em um caso real.

\section{METODOLOGIA}

\subsection{Geometria}

Uma série de 373 imagens de um aneurisma intracraniano sacular humano localizado na artéria cerebral média (MCA) foi cedida pelo hospital Vitória, localizado no Rio de Janeiro. As imagens foram obtidas via angiografia digital, realizada através de cateterismo e injeção de meio de contraste iodado.

Para obtenção da geometria foi utilizado o programa 3DSlicer (www.slicer.org), que permite a reconstrução do volume a partir das imagens da angiografia. O volume gerado foi tratado no software Meshlab, de modo a remover partes indesejadas e porções desconexas da geometria e suavizar as superfícies. A geometria foi então seccionada no programa DesignModeler da ANSYS versão 18.2 de forma a deixar planas suas entradas e saídas. 


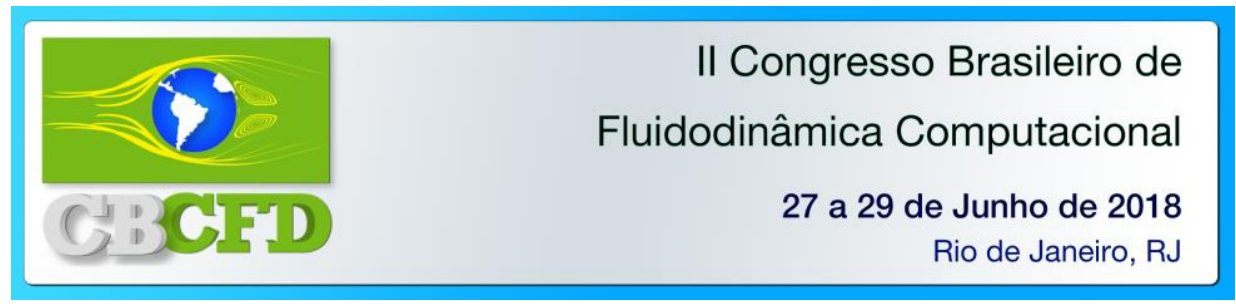

\subsection{Malha}

A malha foi gerada no software Meshing da ANSYS v18.2. Foi utilizada uma malha híbrida composta por elementos prismáticos próximos às paredes e tetraédricos no restante da geometria. A espessura da primeira camada de elementos próximos à parede foi escolhida como $1 \%$ do diâmetro de entrada, com uma taxa de crescimento de 1,1 e um total de 12 camadas concêntricas de elementos. A malha final contém um total de 1.200.971 elementos.

\subsection{Simulações}

As simulações foram realizadas no software FLUENT versão 18.2. O sangue foi considerado um fluido Newtoniano, o que é uma aproximação válida para artérias de grande e médio porte (Xiang et al., 2011) e comumente utilizada em simulações CFD. Foram considerados valores de densidade e viscosidade iguais a $1000 \mathrm{~kg} / \mathrm{m}^{3}$ e $0,004 \mathrm{~kg} / \mathrm{m} . \mathrm{s}$ (Dempere-Marco et al., 2006; Sforza et al., 2016; He et al., 2009).

Sabe-se que as paredes de vasos sanguíneos possuem certa elasticidade e se movem de acordo com as pulsações cardíacas. No entanto, paredes de artérias cerebrais possuem pouca elastina (Jones, 2014) e segundo Dempere-Marco et al (2006), a inclusão de elasticidade nas paredes em simulações resultou em apenas sutis variações em variáveis hemodinâmicas. Assim, a hipótese de paredes rígidas com condição de aderência foi adotada.

Para este trabalho foi utilizado como condição de contorno de entrada um perfil de velocidade periódico e uniforme proposto por He et al (2009) para um aneurisma também localizado na MCA, no qual cada ciclo cardíaco tem a duração de 1 segundo e valores de velocidade variam entre 0,24 e $0,48 \mathrm{~m} / \mathrm{s}$, configurando um escoamento em regime laminar.

Uma vez que não havia dados disponíveis para a pressão nas saídas, foi adotada a condição de contorno de pressão constante igual a $80 \mathrm{mmHg}$, que é a pressão média na MCA de acordo com os limites citados por Jones (2014).

Rodou-se o equivalente a 8 ciclos e os resíduos foram convergidos até $10^{-5}$ utilizando um passo de tempo de 0,001 segundos e 35 iterações por passo de tempo.

\section{RESULTADOS}

Foram observados os principais fatores que se acredita exercerem influência no crescimento e rompimento de aneurismas: tensão cisalhante na parede e perfil do escoamento. Observações de linhas de corrente dentro do aneurisma mostraram a existência de um escoamento complexo, formado por múltiplos vórtices em diferentes instantes de tempo e sem periodicidade, conforme Figura 1 abaixo.

A tensão cisalhante média e máxima na parede do aneurisma apresenta um perfil periódico, porém irregular, variando a cada ciclo. Já a tensão cisalhante média e máxima na parede da artéria apresenta um perfil regular, que se repete a cada ciclo, conforme Figura 2. Os valores encontrados estão de acordo com os reportados na literatura (Sforza et al., 2016). 

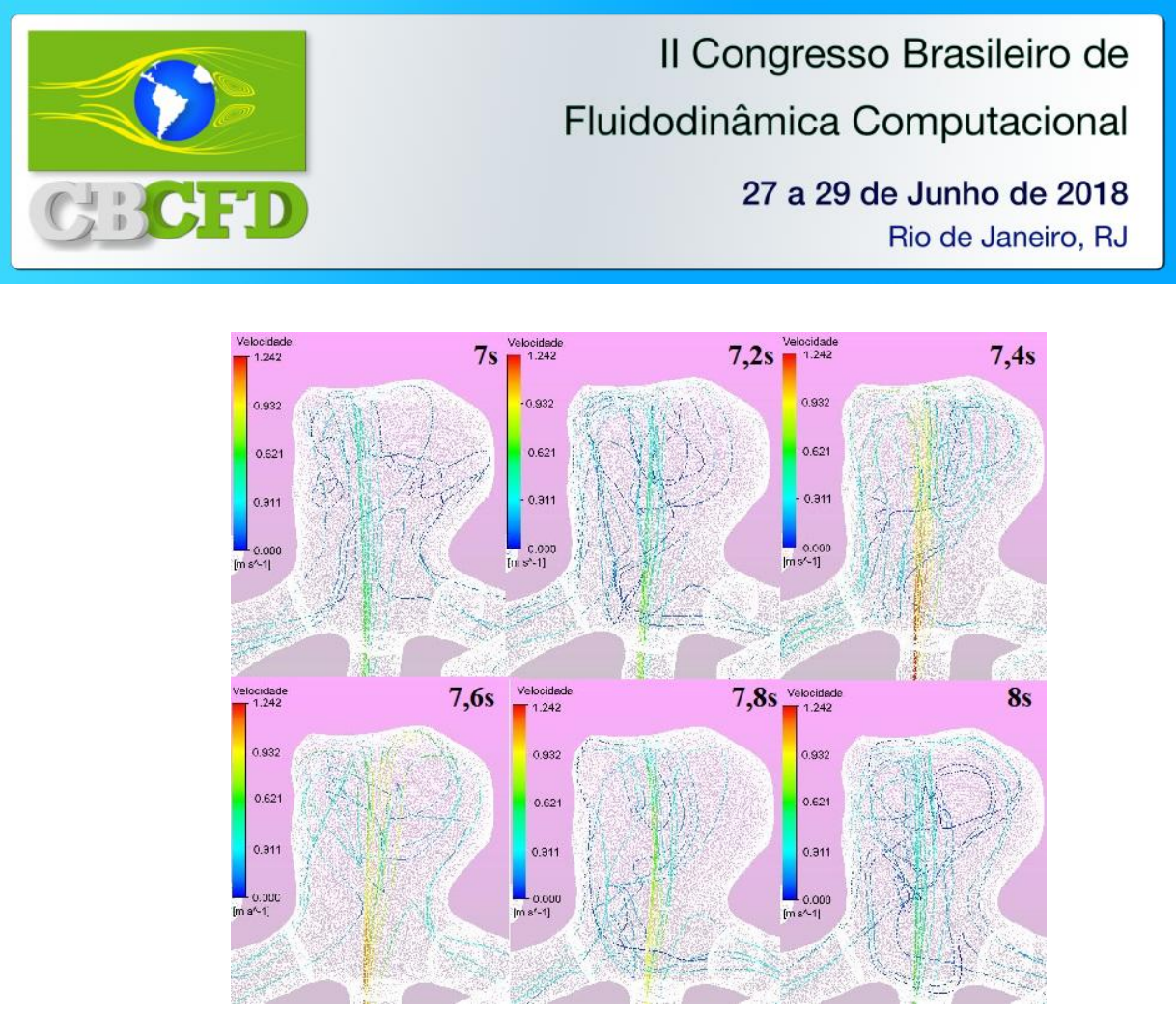

Figura 1 - Linhas de corrente no aneurisma para diferentes instantes de um ciclo.

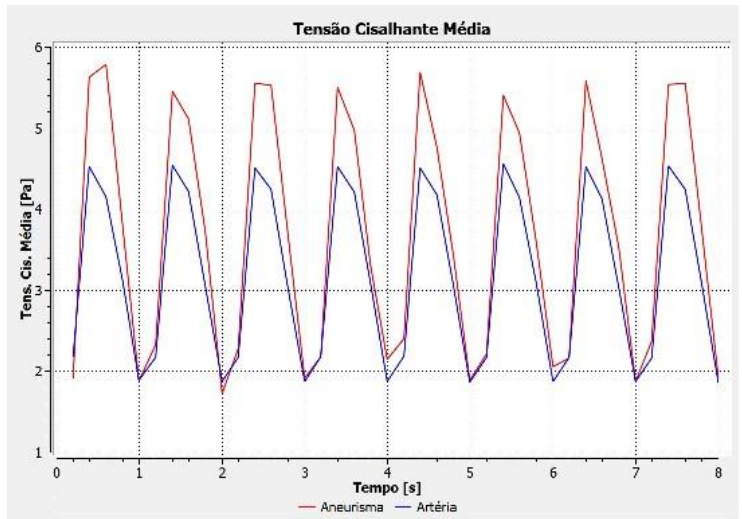

(a)

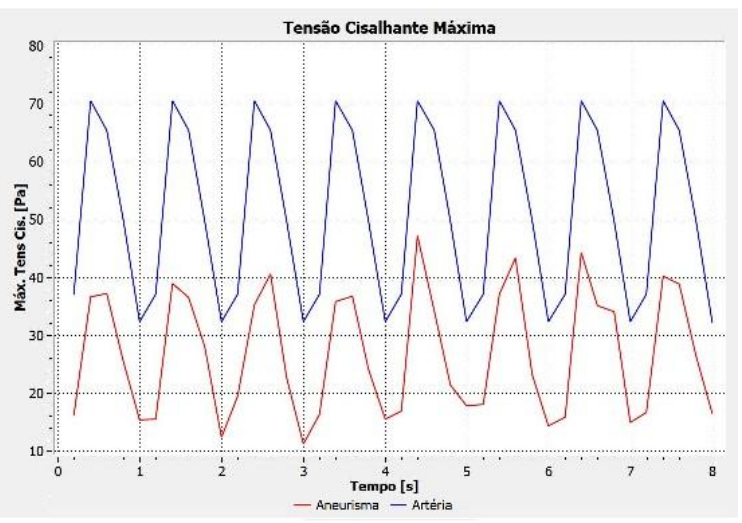

(b)

Figura 2 - Tensão cisalhante (a) média e (b) máxima nas paredes do aneurisma (curva vermelha) e da artéria (curva azul).

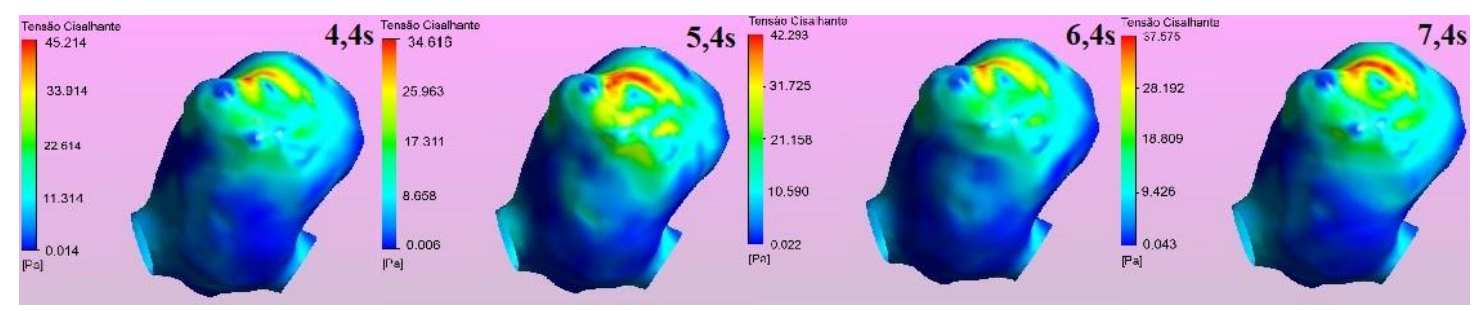

Figura 3 - Área do aneurisma sob máxima tensão cisalhante, em diferentes ciclos

Na Figura 3 pode-se observar os locais onde a tensão cisalhante é máxima nos instantes nos quais o valor máximo é atingido, para diferentes ciclos. Nota-se uma pequena área sob elevada tensão, sempre ao lado esquerdo do aneurisma. 


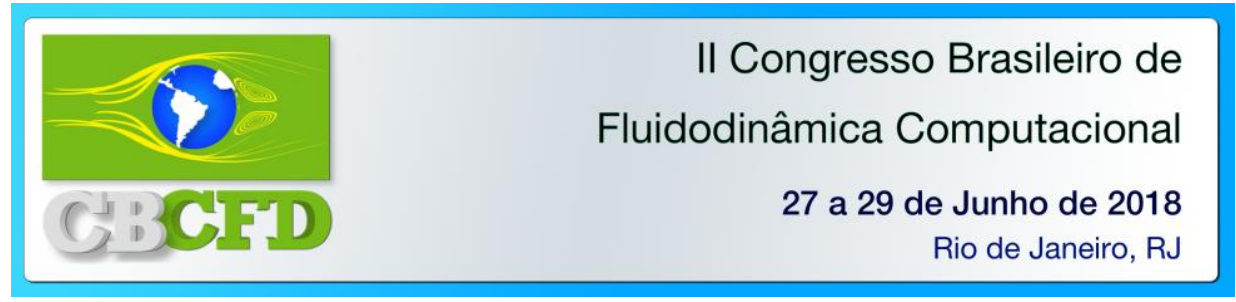

\section{CONCLUSÃO}

Apesar das limitações e simplificações adotadas para as simulações, o perfil de escoamento e a distribuição da tensão nas paredes do aneurisma e da artéria encontrados se mostram coerentes com os relatados pela literatura. Dessa forma, este trabalho reforça que simulações CFD podem contribuir para a área de diagnóstico. No entanto, estudos de mais casos e comparações com dados reais são necessários para que simulações CFD possam vir a auxiliar decisões clínicas.

\section{AGRADECIMENTOS}

Ao Hospital Vitória, do Américas Serviços Médicos do Rio de Janeiro, pelas imagens fornecidas que permitiram a construção da geometria para as simulações.

\section{REFERÊNCIAS}

CIGNONI, P.; CALliERI, M.; CORSINI, M.; DELLEPIANE, M.; GAVONELLI, F.; RANZUGLIA, G.; MeshLab: an Open-Source Mesh Processing Tool Sixth Eurographics Italian Chapter Conference, p. 129-136, 2008.

DEMPERE-MARCO, L.; OUBEL, E.; CASTRO, M.; PUTMAN, C.; FRANGI A., CEBRAL, J.; CFD Analysis Incorporating the Influence of Wall Motion: Application to Intracranial Aneurysms. Med. Image Comput. Comput. Assist. Interv., v. 9, p.438-445, 2006.

FEDOROV, A.; BEICHEL, R.; KALPATHY-CRAMER, J.; FINET, J.; FILLION-ROBIN, JC.; PUJOL, S.; BAUER, C.; JENNINGS, D.; FENNESSY, F.M.; SONKA, M.; BUATTI, J.; AYLWARD, S.R.; MILLER, J.V.; PIEPER S.; KIKINIS, R.; 3D Slicer as an Image Computing Platform for the Quantitative Imaging Network. Magn Reson Imag. v.30, no.9,p. 1323-41, 2012.

KIKINIS, R.; PIEPER, SD.; VOSBURGH, K.; 3D Slicer: A platform for subject-specific image analysis, visualization, and clinical suppoprt. Intraoperative Imaging Image-Guided Therapy, Ferenc A. Jolesz, Editor 3, no. 19, 2014.

HE, X.; DUCKWILER, G.; VALENTINO, D. J.; Lattice Boltzmann simulation of cerebral artery hemodynamics, Comput. Fluids, v. 38, no. 4, p 789-796, 2009.

JONES, Z. R.; The Effect of artery Bifurcation Angles on Fluid Flow and Wall Shear Stress in The Middle Cerebral Artery. Faculty of California Polytechnic State University, 2014.

MUT, F.; LÖHNER, R.; CHIEN A.; TATESHIMA S.; VIÑUELA F.; PUTMAN C.; CEBRAL J.; Computational Hemodynamics Framework for the Analysis of Cerebral Aneurysms. Int. j Numer. Method. Biomed. Eng., v. 27, no. 6, p. 822-839, 2011.

SFORZA, D. M.; KONO, K.; TATESHIMA, S.; VIÑUELA, F.; PUTMAN, C.; CEBRAL, J. R.; Hemodynamics in Growing and Stable cerebral aneurysms, J. Neurointerv. Surg., v. 8, no. 4, p. 407-412, 2016.

XIANG, J.; NATARAJAN, S. K.; TREMMEL, M.; MA, D.; MOCCO, J.; HOPKINS, L. N.; SIDDIQUI, A. H.; LEVY, E. I.; MENG, H.; Hemodynamic-Morphologic Discriminants for Intracranial Aneurysm Rupture, Stroke, v. 42, no. 1, p. 144-152, 2011. 